\title{
Non-painful, acute-onset, unilateral eyelid edema due to wasp sting.
}

\author{
Paul Varner* \\ John J Pershing Veterans' Administration Medical Center, Poplar Bluff, Missouri, USA
}

\begin{abstract}
A 47 year-old male presented with unilateral, non-painful, near-total, non-erythematous left eyelid swelling with anesthesia that developed overnight, approximately 24 hours after a wasp sting within the left eyebrow. The left eye itself remained unaffected. The acute findings completely resolved within four days of the event, mainly with supportive therapy. Hymenoptera venom can have marked neuromuscular effects on the superior eyelid, and clinicians are particularly alerted to the possibility of insect stings involving the supraorbital nerve within the differential diagnosis of non-painful, unilateral eyelid edema.
\end{abstract}

Keywords: acute, unilateral, non-erythematous, non-painful, eyelid edema.

Accepted on March 30, 2017

\section{Introduction}

Providers in pediatric, adult primary care and ophthalmic clinics occasionally examine patients whose presenting symptom is unilateral upper eyelid edema. Such acute-onset presentations in isolation (i.e. no associated eyelid pain, erythema, induration, involvement of ocular or orbital structures, fever, etc.) are both concerning and perplexing to patients; however, often an antecedent event can be identified. This report presents a case for which clinical findings fitting such a description were fully explained by neuromuscular blockade from a wasp stingnot an inflammatory cascade - and which entirely resolved without sequelae. The clinical course followed spontaneous recovery within a few days, and occurred largely independent of pharmacological treatment. The etiology and differential diagnosis of presenting findings, as well as the distinction between inflammatory and non-inflammatory sources for unilateral eyelid edema, are discussed.

\section{Case Report}

A 47 year-old male presented with the complaint of the left eyelids swollen nearly shut upon waking on the day of the initial evaluation. Vision of the left eye seemed unaffected, but only if the patient physically elevated the left upper eyelid (LUL). The left globe itself remained subjectively unaffected. The prodromal history was remarkable for multiple wasp (yellow jacket) stings approximately 24 hours prior to developing the eyelid edema with one site located in the middle of the left eyebrow. There was no eyelid edema immediately after envenomation; however he awoke to find the left eyelids swollen and almost completely closed on the following morning.

External examination revealed near-total closure of the left eyelids, with moderate non-erythematous edema of the LUL in addition to subtle pockets of edema temporally on/below the left lower eyelid (LLL, Figure 1a). There was anesthesia of the entire LUL and limited sensation corresponding to the localized areas of eyelid edema on the LLL. The involved area of anesthesia did not extend to areas surrounding the eyelids (e.g. eyebrow, forehead, upper cheeks, temporal areas, etc.). The patient remained afebrile and had no systemic complaints.
Pupils and extraocular muscle testing were unremarkable, and best-corrected distance visual acuity was $6 / 6$ in both eyes-with mechanical elevation of LUL for entrance testing as the patient was unable to voluntarily elevate the LUL. As measured in the biomicroscope, there was $1 \mathrm{~mm}$ residual palpebral aperture. The left eye itself remained entirely unaffected. The wound site just above the L eyebrow was faintly visible centrally, slightly superior to the hair line; the wasp stinger was not present. Given lack of globe involvement, recommended therapy targeted the eyelid tissue only and consisted of both pharmacological (oral antihistamine to suppress any further histamine-related sequelae) and supportive (cold compresses/eyelid massage to create vasoconstriction to enhance tissue outflow) modes of treatment.

At follow-up examination on the next day, the ptosis had reduced moderately and there was a $6 \mathrm{~mm}$ residual fissure (Figure 1b). There was only minimal anesthesia of the LUL - none for the LLL. The patient indicated that the eyelid edema had begun to resolve, even within hours of the initial ophthalmic evaluation. He measured a $5 \mathrm{~mm}$ palpebral fissure prior to retiring the night before this examination.

The patient reported no subjective improvement the preceding day despite use of oral diphenhydramine (4 mg PO twice) or use of cold compresses/lid massage (approximately $15 \mathrm{mins}$ in duration, also twice) the first morning. Significantly, he noted that the ptosis seemed to improve most after maintaining a vertical body position (i.e. discontinuing bed rest) and especially after going for a walk (60 minutes, minimal elevation in heart rate). The patient was encouraged to continue the physical exercise and to sleep with head elevated in order to determine if relative elevation of the upper body would assist in reducing further the residual eyelid edema. Oral diphenhydramine and cold compresses/lid scrubs were discontinued at that time.

At a final follow-up visit 2 days later (4 days after the insect sting), all residual ptosis, eyelid edema, and lid anesthesia had completely resolved (Figure 1c). The patient had continued daily exercise and sleeping with his head elevated. He had also attempted use of oral NSAIDs (600 mg ibuprofen PO QID, ostensibly to facilitate reduction of inflammation within the 
eyelid), but subjectively reported that this medication offered no additional treatment effect toward resolution of the swollen eyelids. The patient was discharged from further ophthalmic follow-up care.

\section{Discussion}

Hymenoptera (Greek: hymen meaning membrane + "ptera" indicating wings) comprise a ubiquitous order of insects, a small proportion of which have defensive stinging mechanisms including bees, wasps and ants (Figure 2). In humans, stings from these insects induce localized, IgE-mediated Type I hypersensitivity reactions, with various components of the venom inducing histamine and other chemical mediator release from mast cells [1]. The classic signs of inflammation (rubor, calor, dolor and tumor) are all readily apparent at the site of the initial injury as a result of the localized vasodilation and sequelae; however, these effects are not expected to last beyond a few hours. As will be seen, the longer-lasting edema of the LUL for this patient was not the direct result of a Type I sensitivity reaction.

Fortunately, systemic type I hypersensitivity resulting in fatal anaphylaxis occurring from hymenoptera stings is rare- less than one fatality per one million people per year [2] while death from mass envenomation from wasp stings has been estimated to require 20-200 stings [3]. Complex, secondary ophthalmic complications from such sting injuries involving various ocular structures have long been recognized [4].

In broad terms, eyelid edema can be categorized as painful or non-painful in order to facilitate differential diagnosis. The list of painful sources is longer and beyond the scope of this discussion - as are etiologies of bilateral eyelid edema.
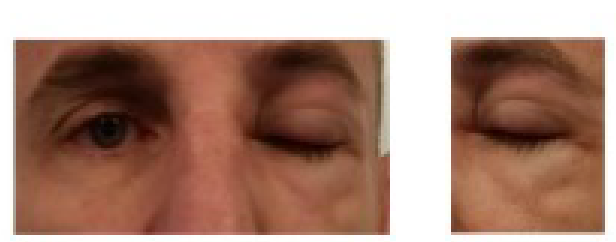

Eissure Sige (vs $12 \mathrm{~mm}$ for $R$ fissure)

Day 1

$$
\begin{array}{ll}
1 \mathrm{am} & 1 \mathrm{~mm} \\
\mathrm{pm} & 5 \mathrm{~mm}
\end{array}
$$

Day 2 am

am 6

pm $10 \mathrm{~mm}$

Day 3 am 11
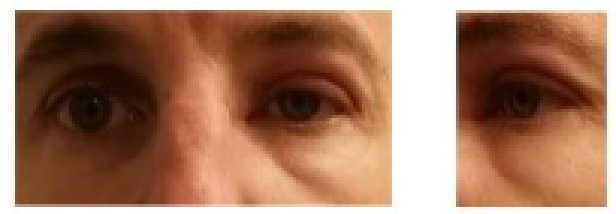

\section{Evelid Anesthesia?}
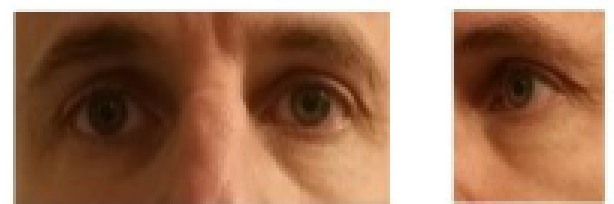
pm 12

$+$

Day 4 am 12 [no residual ptosis]

Figure 1. Frontal and oblique views of left andnexa during follow-up period a)24hours b)48hrs and c)96hrs.

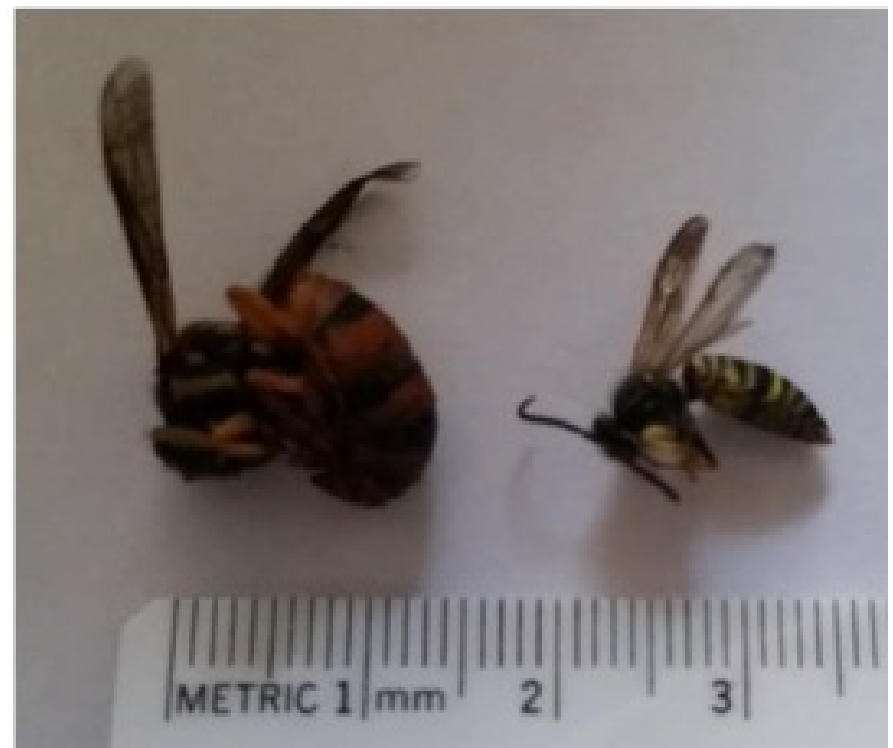

Figure 2. Direct comparision of honey bee(left) to representative wasp(yellow jacket) on right. 
In the absence of chemical mediators for pain; however, the differential diagnosis of unilateral, non-painful eyelid swelling is much more limited. The latter - unilateral, acute-onset, nonpainful, non-erythematous eyelid edema, as in the case of this patient - has been poorly described in the medical literature.

Acute-onset, unilateral eyelid edema in isolation occurs mainly from micro-trauma or allergic reaction. Inadvertent inoculation of the eyelid tissue with any chemical, particulate or environmental contaminant (often involving the same side as the patient's dominant hand) can cause a hypersensitivity reaction that results in localized, unilateral eyelid edema that spares the globe, and often exists with concurrent erythema. The minimal thickness of eyelid tissue ensures that a nominal amount of edema results in a significant physiological effect.

Chronic or recurrent non-painful, unilateral eyelid edema in isolation can be the result of angioedema, neoplasm (eyelid malignancy, periorbital idiopathic inflammation ["pseudotumor"], eosinophilic granuloma, encapsulated infectious/inflammatory cells), or lymphedema in a postoperative setting [5]. Due to limited lymphatics within ocular tissues system [6], such cases do not appear to be related to that system. Cases of chronic or recurrent, asymptomatic eyelid edema typically require neuroimaging and/or eyelid tissue biopsy for definitive diagnosis.

The presence of eyelid edema is an important diagnostic sign in these cases, as sudden-onset upper eyelid ptosis in the absence of other ocular findings - notably swelling — can be an indicator of neurological disease [7]. Regardless, like so many clinical presentations, history is often the key to determining etiology and prognosis. "Idiopathic" cases should be viewed with caution, although patients may not always clearly associate antecedent events with ophthalmic consequences.

On the microscopic level, the non-painful eyelid edema that developed in this case was the result of fluid accumulation in the fascial spaces of the eyelids. The retention of interstitial body fluid within the eyelid tissue was exacerbated during the hours of sleep, once gravity was no longer present to assist in the mechanical evacuation of fluid, and was dampened by concurrent neurological block.

Wasp venom is a known neuromuscular blocking agent [8] its introduction near the supraorbital nerve of this patient wrought both neurological and muscular effects. Impairment of the normal, neurological feedback loop compromised normal fluid exchange within the eyelid tissue, which was highly exacerbated by horizontal body positioning during sleep. The effected muscular block was evidenced by the patient's inability to voluntarily elevate the LUL. Fortunately - as with neuromuscular blocking agents - the pharmacological effects of the wasp venom were temporary and all physiological integrity was restored within four days of the sting.

These pharmacological and physiological events support the patient's report that elevation of the affected tissue and physical exercise seemed to provide more relief than did various pharmaceutical agents combined with cold compresses. Thus the functio laesa encountered by this subject was pharmacological — and not inflammatory — in origin.

As final considerations: the use of anti-venom therapies seems unwarranted for largely self-limited findings as in this case; and resolution of clinical findings within one week would appear to be the expected result for this and similar cases [9].

In the absence of a contributing ophthalmic history, or nonresolution of the eyelid edema within one week - or in the presence of recurring eyelid edema-MRI neuroimaging may be required to rule out neoplasm or myositis and eyelid tissue biopsy may be indicated. Those patients may need oral corticosteroid therapy in order to reduce clinical findings

\section{Conclusion}

While the differential diagnosis for unilateral, non-painful eyelid edema is not lengthy, careful attention to the case history should go far toward understanding the source of the eyelid swelling. The presence of eyelid anesthesia will assist in the establishment of a neuromuscular blocking etiology for the eyelid edema.

Patients can be reassured that clinical findings are typically selflimited, resolve within a few days of injury, and do not result in long-term sequelae. Sleeping with the head elevated combined with non-vigorous physical exercise may provide better relief than various oral pharmaceutical agents due to the nature of the neuromuscular blockade involving the supraorbital nerve.

In addition to micro-trauma and allergy-more traditional inflammatory cascade events - an insect-vector, pharmacological source for unilateral non-painful eyelid edema must be considered In this case, the clinical findings were the result of regional neuromuscular block of the supraorbital nerve via wasp venom.

\section{Acknowledgment}

The author would like to thank Antonia Varner for her assistance in the preparation of the figures.

\section{Disclosure}

The views expressed in this article are those of the author and do not necessarily represent the positions of the Department of Veterans Affairs. The author reports no proprietary or commercial conflict of interest for any product mentioned or concept discussed in this article.

\section{References}

1. Riches, H.R.C. (1982). Hypersensitivity to Bee Venom. Bee World, 63(1), 7-22.

2. Bilò, M.B. (2011). Anaphylaxis caused by Hymenoptera stings: from epidemiology to treatment. Allergy, 66(Suppl. 95), 35-37.

3. Vetter, R.S., Visscher, P.K., Camazine, S. (1999). Mass envenomations by honey bees and wasps. West $\mathrm{J} \mathrm{Med,}$ 170(4), 223-227.

4. Duke-Elder, S. (1974). In System of Ophthalmology, ed S. Duke-Elder, Vol VIII, pp 201-205. The C.V. Mosby Company: St Louis.

5. Wong, B.J., Hong, B.K., Samrao, D., Kim, G.H., \& Rao, N.A. (2014). A 49-year-old man with unilateral, nontender left eyelid swelling. Digit J Ophthalmol, 20(1), 15-19. 
6. Nakao, S., Hafezi-Moghadam, A., Ishibashi, T. (2012). Lymphatics and lymphangiogenesis in the eye. J Ophthalmol, 2012:783163.

7. Court, J.H., Janicek, D. (2014). Acute unilateral isolated ptosis. BMJ Case Rep, 2015 Jan 5.

8. Piek, T., Mantel, P., Engels, E. (1971). Neuromuscular block in insects caused by the venom of the digger wasp Philanthus Triangulum F. Comp Gen Pharmacol, 1971, 2(7), 317-331.

9. Rayward, O., Vallejo-Garcia, J.L., Moreno-Martin, P., \& Vano-Galvan, S. (2013). Diagnostic puzzler: acute eyelid edema. J Fam Pract, 62(5):E 1-3.

\section{*Correspondence to:}

Paul Varner

John J Pershing Veterans' Administration Medical Center

USA

Tel: +1 5737784303

E-mail: paul.varner@va.gov 